\title{
The impact of health education on treatment outcomes in heart failure patients
}

\author{
Natalia Alicja Świątoniowska-Lonc 1,B-D, Agnieszka Sławuta, ${ }^{2, B-D}$, Krzysztof Dudek ${ }^{3, B, C}$, \\ Katarzyna Jankowska ${ }^{4, B, D}$, Beata Katarzyna Jankowska-Polańska, ${ }^{1, A, E, F}$ \\ ${ }^{1}$ Faculty of Health Sciences, Wroclaw Medical University, Poland \\ ${ }^{2}$ Department of Internal Medicine, Occupational Diseases, Hypertension, and Clinical Oncology, Wroclaw Medical University, Poland \\ ${ }^{3}$ Department of Logistic and Transportation Systems, Wroclaw University of Science and Technology, Poland \\ ${ }^{4}$ Department of Conservative Dentistry and Pedodontics, Wroclaw Medical University, Poland \\ A - research concept and design; $\mathrm{B}$ - collection and/or assembly of data; $\mathrm{C}$ - data analysis and interpretation; \\ $D$ - writing the article; $E$ - critical revision of the article; $F$ - final approval of the article
}

\section{Address for correspondence}

Natalia Alicja Świątoniowska-Lonc

E-mail: natalia.swiatoniowska@student.umed.wroc.pl

\section{Funding sources}

None declared

Conflict of interest

None declared

Received on September 20, 2019

Reviewed on October 28, 2019

Accepted on December 5, 2019

Published online on April 29, 2020

\section{Abstract}

Background. In 2016 heart failure (HF) affected between 600,000 and 700,000 people in Poland being one of the most common causes of hospitalization and death. Health education is an elements of patient treatment aimed at improving the level of self-care and adherence to the treatment recommendations.

Objectives. To perform a systematic review and meta-analysis of the available literature in order to determine the role of health education in $\mathrm{HF}$ treatment and its impact on outcomes in patients with chronic HF.

Material and methods. A search was performed in the MEDLINE, PubMed and Scopus databases from January 2010 to January 2019 for the impact of health education on treatment outcomes in HF patients.

Results. A total of 16 studies from 12 countries on 5 continents were analyzed. The meta-analysis focused on the impact of education on outcomes in 944 study group patients. We found that the overall impact of education on outcomes was positive (+1 standard deviation (SD); $95 \%$ confidence interval $(95 \%(\mathrm{Cl})>0$ ). After education was provided, the target patients improved in terms of self-care (mean change $(M C)=13.49$; $\left.p=0.003 ;\left.\right|^{2}=99.47 \%\right)$. Self-care also improved in the controls, but the improvement was less marked $\left(M C=9.56 ; p=0.001 ; I^{2}=98.33 \%\right)$. No impact of education on quality of life (QoL) was confirmed $(95 \% \mathrm{Cl}=0)$.

Conclusions. The greatest benefit of education is seen in terms of adherence to pharmaceutical treatment and self-care, while QoL was not associated with education.

Key words: treatment, education, outcome, heart failure

Cite as

Świątoniowska-Lonc NA, Sławuta A, Dudek K, Jankowska K, Jankowska-Polańska BK. The impact of health education on treatment outcomes in heart failure patients. Adv Clin Exp Med. 2020;29(4):481-492. doi:10.17219/acem/115079

DOI

10.17219/acem/115079

Copyright

Copyright by Author(s)

This is an article distributed under the terms of the

Creative Commons Attribution 3.0 Unported (CC BY 3.0)

(https://creativecommons.org/licenses/by/3.0/) 


\section{Introduction}

Depending on the diagnostic criteria used and the population studied, worldwide heart failure (HF) incidence is $100-900$ cases per 100,000 person-years. Its global prevalence is estimated at 37.7 million cases. In developed countries, HF affects $1-2 \%$ of the adult population. ${ }^{1}$ The number of HF patients in the USA currently stands at 5.8 million, and is expected to exceed 8 million by the year 2030. One in 5 individuals can be expected to develop HF at some point in their life. ${ }^{2}$ Heart failure is a clinical syndrome associated with frequent hospitalizations and complex treatment regimen. Despite advances in HF diagnosis and treatment, $50 \%$ of patients globally are rehospitalized within 6 months of discharge, and approx. 300,000 die annually due to HF decompensation. ${ }^{3,4}$ Nonadherence to treatment is the primary cause of mortality and rehospitalization in HF patients. ${ }^{5}$ Out of these rehospitalizations, between $1 / 3$ and $1 / 2$ may be preventable. ${ }^{6}$

Heart failure treatment guidelines produced by scientific societies emphasize the role of multidisciplinary care, comprising self-care, compliance with treatment and follow-up visits as major factors that improve patient outcomes. In HF treatment, lifestyle changes, self-care, health-promoting practices, and symptom monitoring and management are as important as pharmaceutical treatment. The objective of the entire treatment regimen is to improve the patient's physical fitness and quality of life (QoL), prevent rehospitalization and reduce overall mortality. ${ }^{7}$ Considering the complexity of HF and its treatment, relevant knowledge is a major factor in effective therapy. Education is therefore a component of the treatment process in $\mathrm{HF}$, and its main areas include preparing the patient for cooperation with the treatment team, flexible dosages of diuretics, adherence to a low-sodium diet, exercise, daily weighing, fluid intake restrictions, recommended vaccinations, monitoring symptoms, and reacting to exacerbations.

Effective education programs for HF patients have been shown to enhance the patients' knowledge, improve their self-care capabilities, reduce the number of hospitalizations, and improve outcomes and QoL. ${ }^{8}$ In HF patients, self-care has been defined as a naturalistic decision-making process with 2 components: self-care maintenance and self-care management.

Traditional patient education focuses on providing learning material. However, this form of education is often insufficient to influence patients' self-care behavior. The available study results remain unsatisfactory, demonstrating little impact of education on better HF patient outcomes. The overall objective of the implemented educational projects is the development of the patients' selfmanagement skills and capabilities of improving their own QoL. The studies that are available do not describe education as a specific intervention; it is typically part of the HF management program. The benefits of education are well understood, but it is difficult to tell which education method is the most effective.

According to the World Health Organization (WHO), health education is a process consisting of planned learning and communication opportunities that improve knowledge on health, health-related skills and life skills conducive to health in individuals and communities. Patient counseling is based on behavioral therapy and social cognitive therapy. ${ }^{9}$

The available study results highlight the importance of motivational education. Motivational conversations represent an individual-focused approach, in which thoughtful listening is used rather than confrontation. Medical professionals preparing a patient for performing self-care should use the "education through motivation" approach, as this form of education has been found to be more effective than other methods. Patients demonstrate a willingness to change their behavior and employ strategies aimed at actively taking responsibility for their own health. Education is even more effective if the process is personalized, and patients receiving individualized education show more clinical improvement than those under routine care..$^{10,11}$

Publications on the effectiveness of education and its association with the effectiveness of $\mathrm{HF}$ treatment remain scarce. Authors with an interest in HF report a need for further studies that would evaluate the effectiveness of education with regard to secondary outcomes in HF patients.

\section{Objectives}

The aim of the study was to perform a systematic review and meta-analysis of the available literature in order to determine the role of health education in HF treatment and its impact on outcomes in patients with chronic HF.

\section{Methods}

The present study investigated the impact and effectiveness of health education in any form with regard to chronic HF treatment outcomes. The outcomes analyzed include QoL, compliance, self-care behavior, and rehospitalization.

\section{Search strategies}

The MEDLINE, PubMed and Scopus databases were searched using the following keywords: "education", "chronic heart failure", "self-care" OR "self-management" OR "persistence” OR "adherence" OR "compliance” AND "outcomes". Additional criteria were used to restrict the search scope to research papers published in English between January 2010 and January 2019. Review papers, duplicates and other meta-analyses were excluded. 
The final material consisted of 17 papers. Subsequent analyses were performed using the Cochrane Review Manager guidelines. ${ }^{12}$ Adherence and compliance, self-care, self-management, QoL, and HF-associated rehospitalizations were understood as secondary outcomes. The search procedure is presented in detail in Fig. 1.

\section{Description of the studies included}

The 17 studies included in the meta-analysis were performed in 12 countries on 5 continents. The metaanalysis focused on the impact of education on outcomes in 944 HF patients (out of 3,567 patients in total). Sixteen of the studies analyzed were randomized. The most common randomization procedures included the use of a computer-generated sequence (5 studies) or a randomization center office (2 studies). In 3 studies, randomization was performed by nurses, and in 2 by physicians. One study used sealed envelopes, and another used the website www. randomization.com (Table 1).

Twelve of the studies enrolled hospitalized patients following an acute event, and 5 enrolled outpatients. Male patients comprised $57.5 \%$ of the study groups. The mean patient age was $66.9 \pm 13.2$ years. Exclusion criteria were as follows: cognitive impairment (9 studies), severe comorbidities (11 studies), kidney disease (3 studies), life expectancy up to 6 months ( 1 study) or up to 12 months ( 3 studies), and surgery within 6 months preceding the study (2 studies).

\section{Questionnaires used to assess the quality of life}

The following questionnaires were used:

1. The Kansas City Cardiomyopathy Questionnaire (KCCQ) is a 23-item (15 questions), self-administered, disease-specific instrument that quantifies 6 domains (scales) and 2 summary scores of the patient's health status. The 6 domains are physical limitations, symptom score, symptom change, self-efficacy, social interference, and QoL. The 2 summary scores are labelled clinical summary scores and overall summary scores. All scale scores are transformed to $0-100$ scale, in which a higher score indicates a better health-related quality of life (HRQoL).

2. The Iranian Heart Failure QoL questionnaire (IHF-QoL), a 16-item questionnaire in which questions 1-4 and 6 measure the symptoms of the disease and their severity (score $5-20$ ); question 7 is about physical limitations in performing activities (score 5-15); questions 8, 10, 12, and 13 are about social interference (score 4-12); questions 5, 9 and 11 are about psychological conditions (score 3-10); questions 14 and 15 are about knowledge and self-efficacy (score 2-6); and question 16 is about the patient's life satisfaction (score 1-3). The questions are answered using a 3- or 4-point Likert scale. The total QoL scores range from 15 to 63 and are calculated by summing the scores of the questions. Higher scores indicate a higher QoL in total and in each dimension.

3. The Minnesota Living with Heart Failure Questionnaire (MLHFQ) has 21 items, which cover the physical

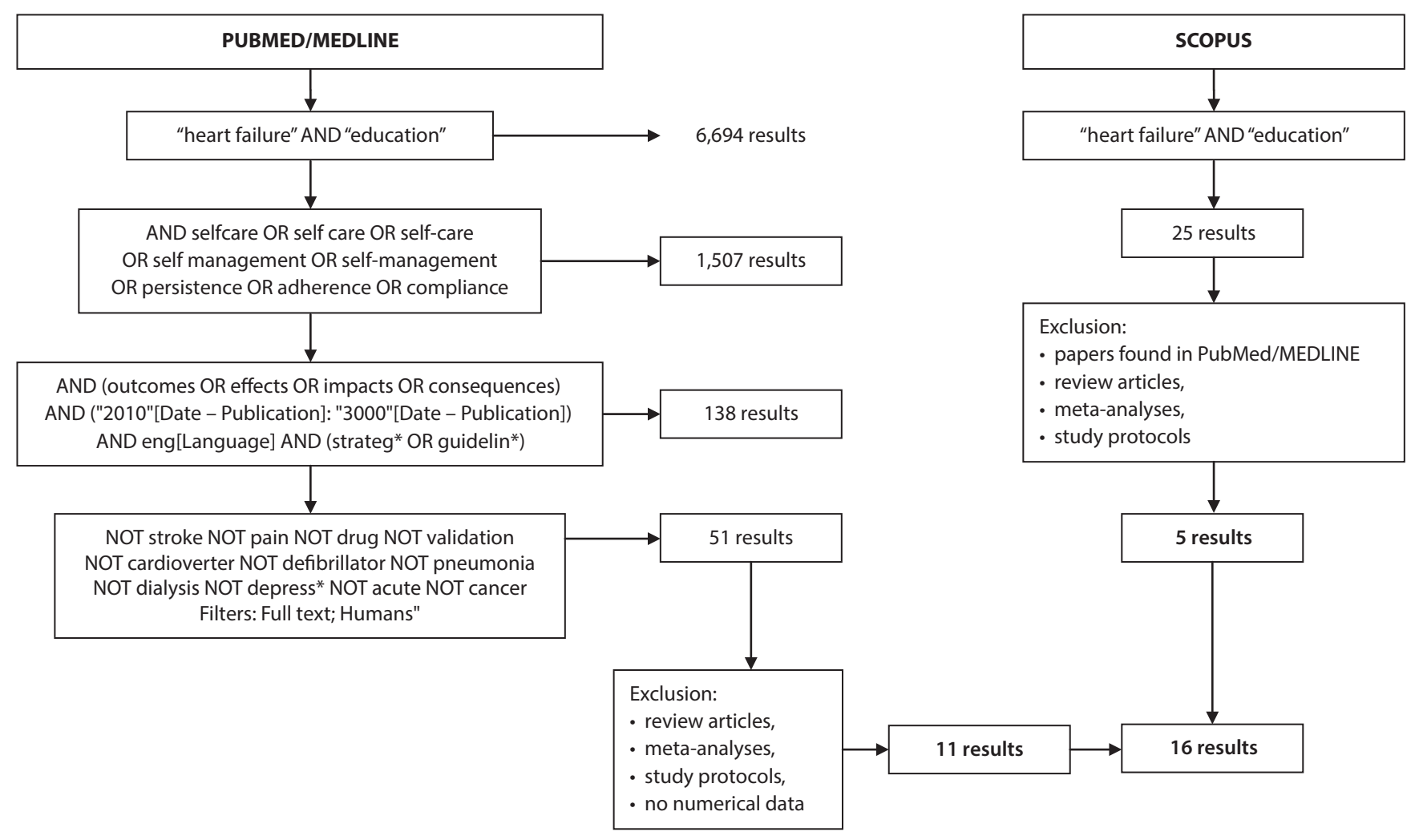

Fig. 1. Study flow diagram 
Table 1. Summary of studies of the impact of education on outcomes in HF

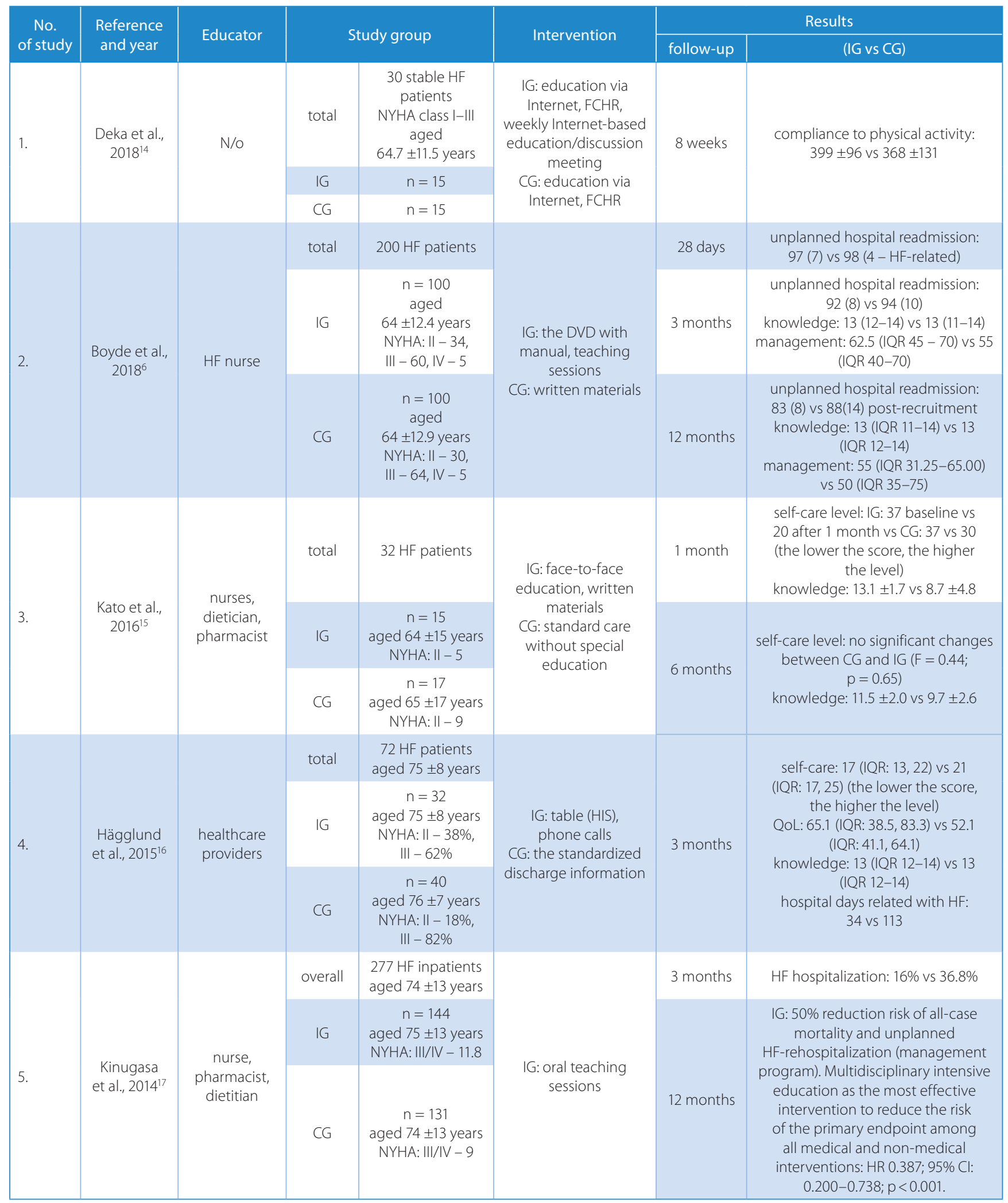


Table 1. Summary of studies of the impact of education on outcomes in HF - cont.

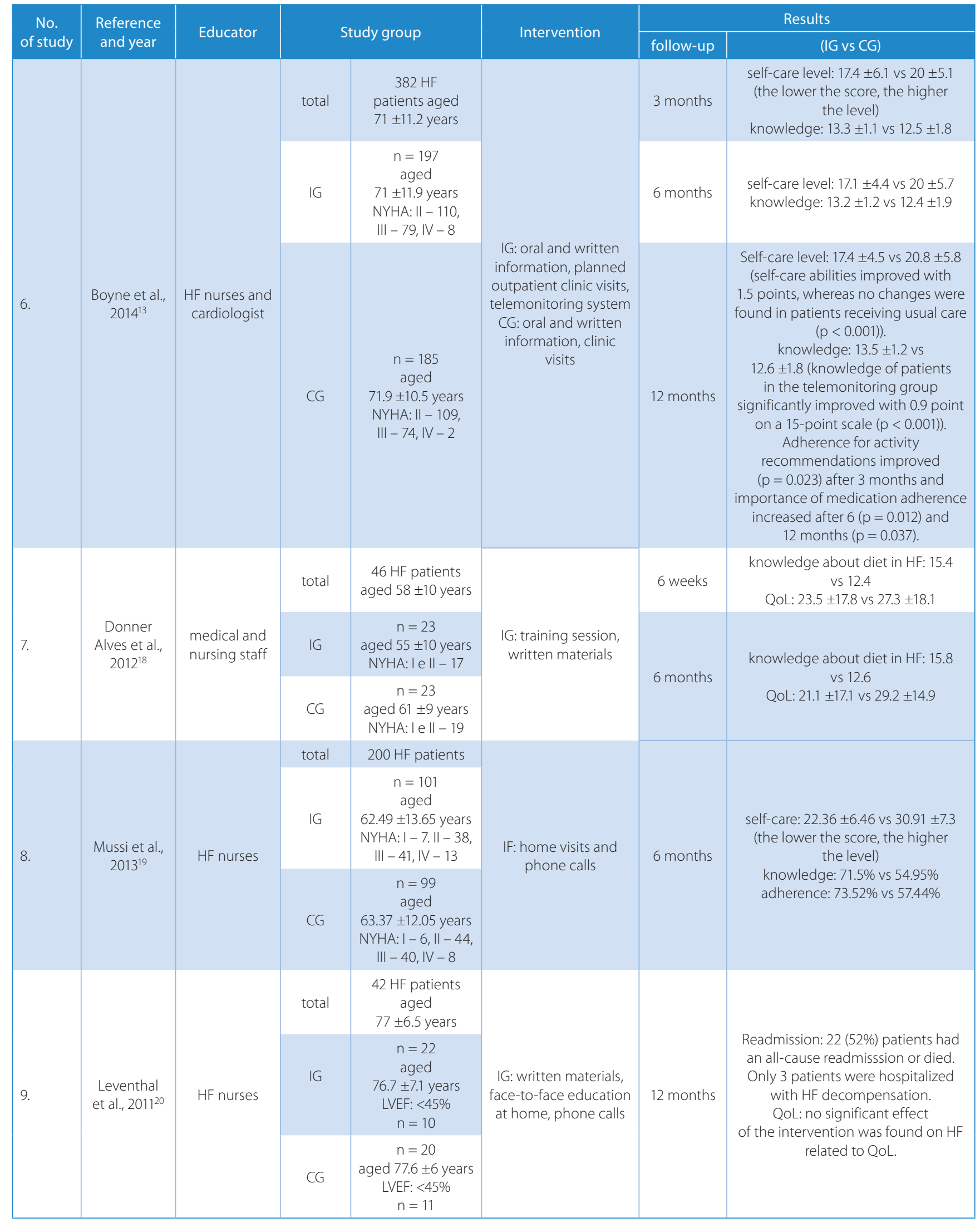


Table 1. Summary of studies of the impact of education on outcomes in HF - cont.

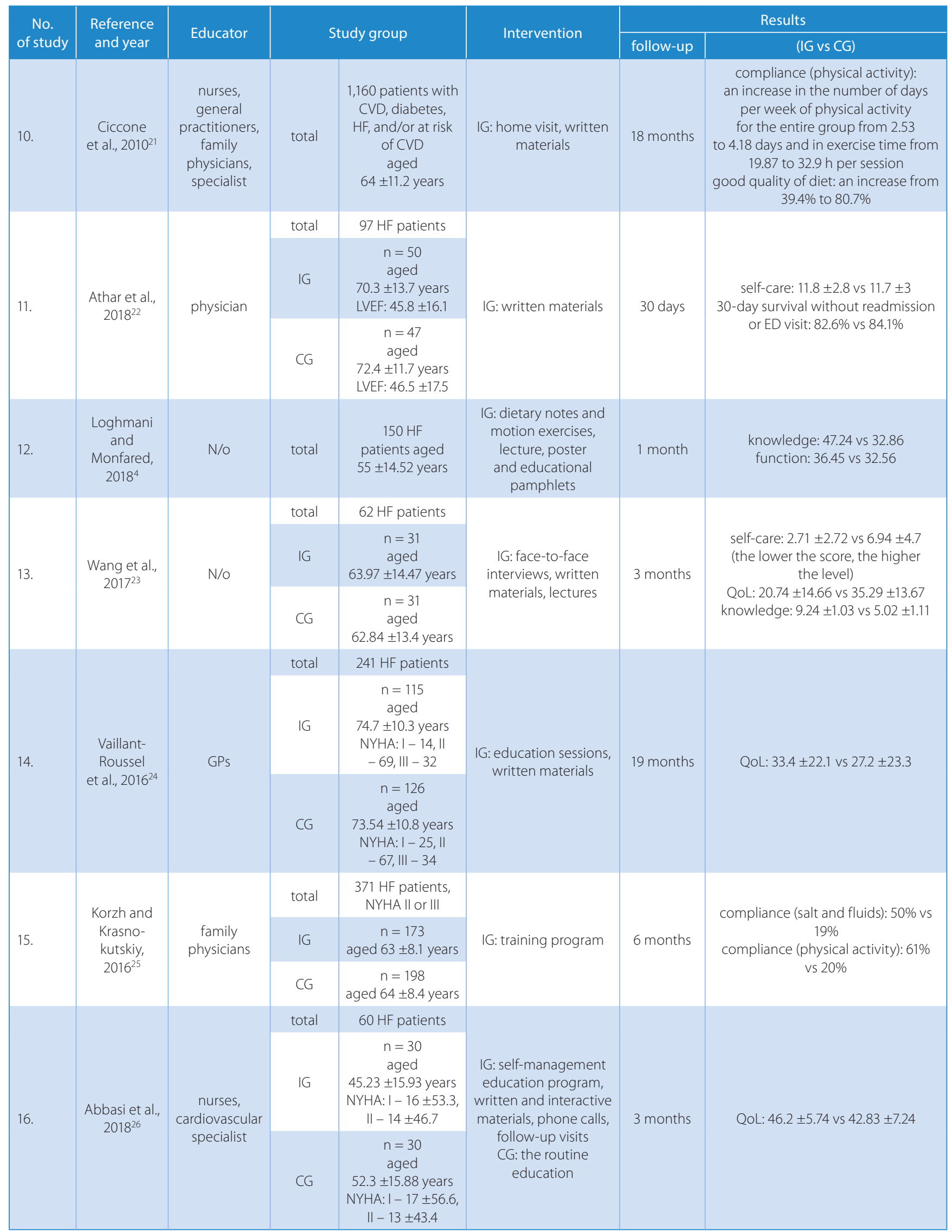

N/O - no information; NYHA - New York Heart Association; GP - general practitioner; CG - control group; IG - intervention group; FCHR - the Fitbit Charge HR; LVEF - left ventricular ejection fraction; CVD - cardiovascular disease; HF - heart failure; ED - emergency department; IQR - interquartile range; 95\% Cl - 95\% confidence interval; HR - hazard ratio; QoL - quality of life. 
dimension (8 items), emotional dimension (5 items) and additional dimension (8 items). Each item is scored from 0 to 5 , and the total score ranges from 0 to 105 . A lower score indicates better QoL.

\section{Statistical analysis}

In the case of continuous-scale studies (comparing means between cases and controls), the meta-analysis used Hedges' $g$ to calculate the standardized mean difference in the fixed effects model. Heterogeneity statistics were subsequently used to calculate the total standardized mean difference in the random effects model.

In each meta-analysis, the mean change (MC) in outcomes measured before and after the educational intervention was used. Positive MC values indicate higher outcome scores after the educational intervention (i.e., improved outcome compared to that measured before the intervention), while negative $\mathrm{MC}$ values indicate higher outcome scores before the educational intervention (i.e., deteriorated outcomes).

As the authors of the papers included in the meta-analysis used a variety of questionnaires, all the scales were converted into a $0-100$ scale using the following formula:

$$
\frac{x-\min }{\max -\min } \times 100
$$

where min and max stand for the minimum and maximum number of points that could be obtained in a given questionnaire.

Additionally, if a higher score represented a lower level of self-care/knowledge/QoL, the scale was reversed for the purpose of this meta-analysis.

An $I^{2}$ parameter was used to assess the degree of heterogeneity. The $\mathrm{I}^{2}$ value was calculated as $\mathrm{I}^{2}=100 \% \times(\mathrm{Q}-\mathrm{df}) / \mathrm{Q}$, where $\mathrm{Q}$ is the statistics of Cochran heterogeneity and $\mathrm{df}$ is degrees of freedom. The $\mathrm{I}^{2}$ values are percentages. The 95\% uncertainty intervals were calculated according to the proposals set forth by Higgins and Thompson. ${ }^{27}$

\section{Results}

\section{Educational interventions}

The studies analyzed included the following types of educational interventions:

1. Training/education sessions for patients (educational sessions: 8 studies; written materials or DVDs: 8 studies; online learning: 2 studies). The sessions included such topics as diet, fluid restriction, exercise, pharmaceutical treatment, and knowledge related to HF. Educational sessions were provided by specialized nurses, physicians, dietitians, pharmacists, physical therapists, and social workers. A variety of methods and materials were used, such as booklets, discussion services, brochures, lectures, newsletters, multimedia presentations, as well computer programs and software.

2. Patient reminder systems (phone calls: 3 studies; home visits: 3 studies). Regular telephone calls or home visits were conducted by HF nurses and/or physicians. During the phone calls, self-care and the application of knowledge provided in the sessions were discussed. Home visits involved physical, psychosocial and environmental evaluations; repeat education to improve self-care; and setting individual treatment objectives.

3 . Telemonitoring systems ( 2 studies), involving 2 methods. In the first method, patients were presented with preset dialogs and questions about their symptoms, knowledge and behavior on a daily basis, and provided answers using touch keys. In the event of any problems, an HF nurse contacted the patient. ${ }^{13}$ In the other method, Fitbit Charge HR (FCHR), information on step count, heart rate, and minutes of exercise were tracked, recorded and transmitted in real time. Exercise sessions could also be recorded using a built-in stopwatch. ${ }^{14}$

\section{The effectiveness of the interventions}

\section{The impact of education on knowledge about the illness}

The impact of education on patients' knowledge about the illness was measured in 7 studies (Fig. 2). In the metaanalysis, the total effect of education was positive (+1 standard deviation (SD) to the right of 0 , where 0 indicates no impact), and the lower bound of the $95 \%$ confidence interval $(95 \% \mathrm{CI})$ was greater than 0 .

\section{The impact of education on adherence and compliance}

Adherence and compliance in HF patients was evaluated in 7 studies (Fig. 3). Due to the lack of control groups

Kato 2016

Hägglund 2015

Wang 2017

Loghmani 2018

Boyde 2018

Boyne 2014

Mussi 2013

Total (fixed effects) Total (random effects)

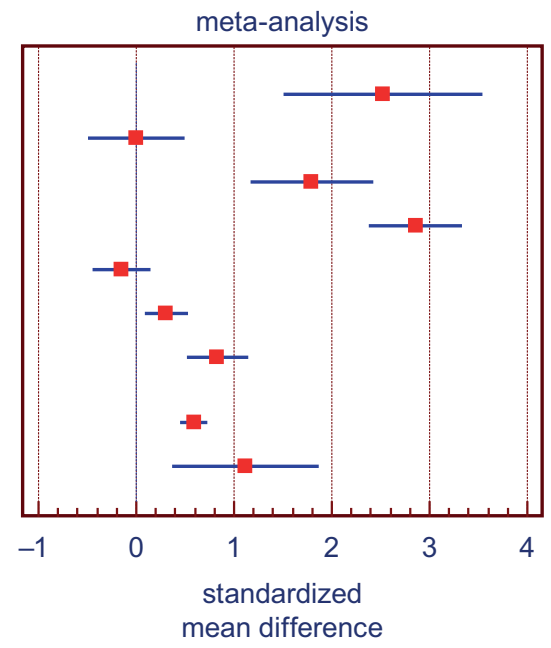

Fig. 2. The impact of education on knowledge (means and 95\% Cl values) 


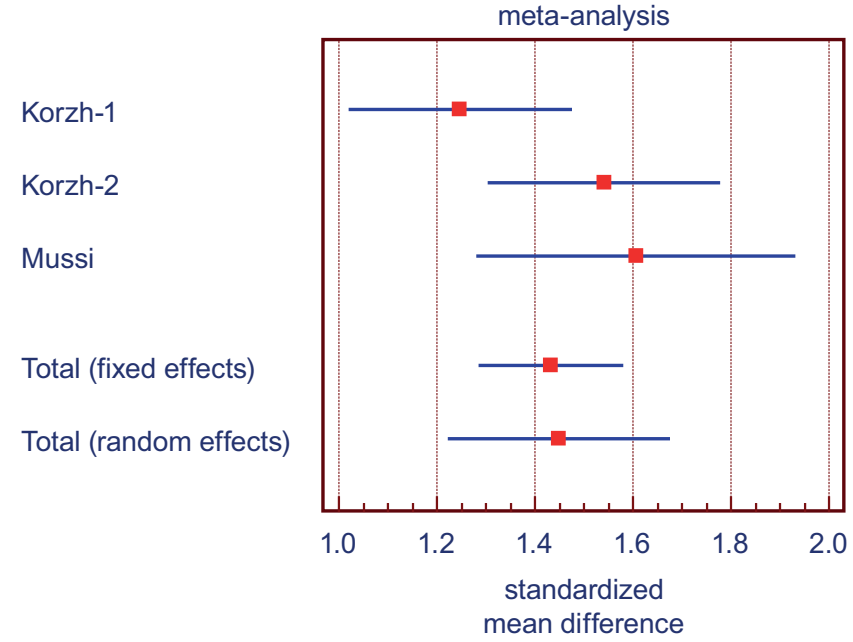

Fig. 3. The impact of education on adherence and compliance (means and $95 \%$ Cl values)

and discrepancies in the reported data, only 3 papers were ultimately included in this part of the meta-analysis. The total effect of education was positive (over +1 $\mathrm{SD}$ to the right of 0 , where 0 indicates no impact), and the lower bound of the $95 \% \mathrm{CI}$ was greater than 0 (the left end point of the horizontal segment).

\section{The impact of education on self-care}

\section{Patients who received education}

The impact of education on self-care in HF patients was evaluated in 6 studies (Fig. 4). The meta-analysis result was statistically significant $(\mathrm{p}=0.003)$, which means that evidence of significant changes in self-care was found among those patients who received education. An MC value of 13.49 indicates that the mean increase in the self-care score resulting from educational interventions was 13.49 points. The test for heterogeneity demonstrated considerable heterogeneity of the studies analyzed $(\mathrm{p}<0.001)$, and therefore these results were obtained using the random effects model. The heterogeneity coefficient was $\mathrm{I}^{2}=99.47 \%$.

The forest plot shows a total MC value of 13.49 points from the meta-analysis $(95 \% \mathrm{CI}=4.52-22.46)$. This means that after

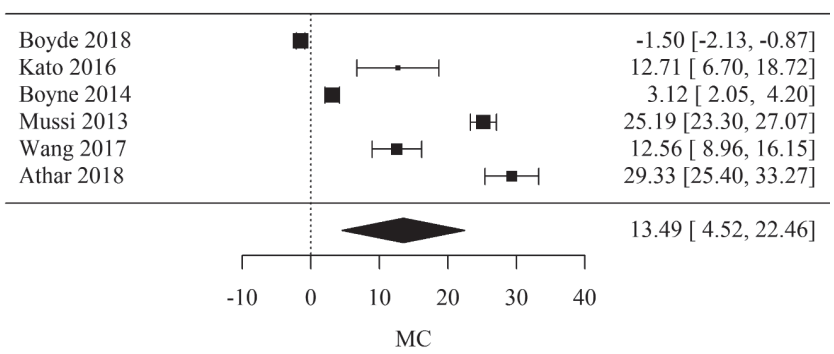

Fig. 4. The impact of education on self-care in the group of patients who received education

$M C$ - mean change. educational interventions, the targeted patients in the studies improved their self-care scores by a mean of 13.49 points. Self-care improved after education in all the studies included in the meta-analysis except for Boyde et al. ${ }^{6}$

\section{Patients who did not receive education}

Significant evidence of changes in the self-care level was found among patients who were not targeted by the educational interventions (Fig. 5). An MC value of 9.56 indicates that after the period designated for the educational intervention, these patients scored, on average, 9.56 points higher on the self-care scale. The test for heterogeneity demonstrated considerable heterogeneity of the studies analyzed ( $\mathrm{p}<0.001)$, and therefore these results were obtained using the random effects model. The heterogeneity coefficient was $\mathrm{I}^{2}=98.33 \%$.

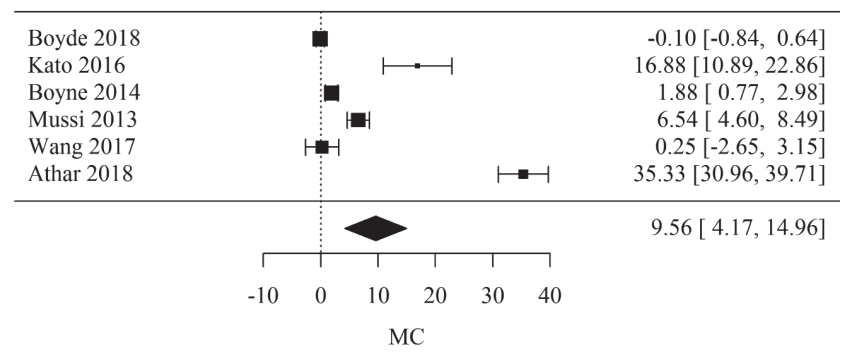

Fig. 5. Changes in self-care in the group of patients who did not receive education

\section{The impact of education on QoL}

The impact of education on QoL in HF patients was evaluated in 6 studies (Fig. 6) on the basis of the results of questionnaires evaluating QoL at the baseline and the follow-up points. Due to the lack of a control group in Donner Alves et al. and Leventhal et al., only 4 studies were included in the further analysis. ${ }^{18,20}$ No impact of education on QoL

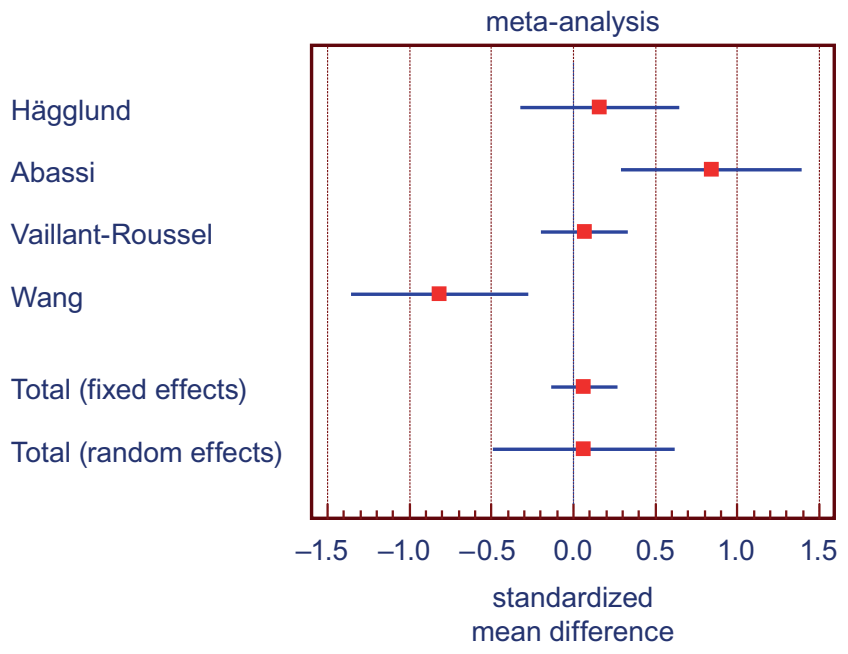

Fig. 6. The impact of education on QoL (means and 95\% Cl values) 
was found in the studies analyzed. In all the studies, 95\% CI was 0 , except for the study by Wang, who found a negative impact of education on QoL. ${ }^{23}$

\section{The impact of education on HF-associated rehospitalizations}

The impact of education on the number of HF-associated rehospitalizations in HF patients was evaluated in only 3 studies. Hägglund et al. measured the number of days between subsequent hospitalizations for HF (intervention group 113 days vs control group 64 days). ${ }^{16}$ Leventhal et al. reported the number of HF-related readmissions. ${ }^{20}$ In a study by Vaillant-Roussel, 18 patients in the intervention group and 22 patients in the control group had hospitalizations for CHF decompensation. ${ }^{24}$ Due to the lack of complete data, a meta-analysis could not be performed for this variable.

\section{Discussion}

Factors interfering with HF care include insufficient knowledge on the part of the patients, which results in inadequate self-control and self-care, as well as a lack of a comprehensively integrated care system. Therefore, a new model of care for HF patients is required - one that would include education focused on the implementation of standards to lower the rate of rehospitalizations and mortality, reduce costs, and improve the QoL of patients and their families.

The purpose of the present meta-analysis was to investigate the impact and effectiveness of health education in any form with regard to chronic HF treatment outcomes. The outcomes that were analyzed included QoL, compliance, self-care behavior, and rehospitalization. This metaanalysis confirmed that studies on the role of education for HF patients and its impact on outcomes remain insufficient. Knowledge and education are presented by many authors as important factors in HF treatment, but the studies published report few actual educational interventions and their effects. Out of all the available publications, only 16 met the selection criteria for the present meta-analysis. The meta-analysis only included studies that compared the impact of education using an intervention group and a control group, which makes it unique.

Through the meta-analysis, we confirmed the significance of education in patients with HF and its beneficial role in outcomes. In many cases, patients after an educational intervention experienced a greater improvement of outcomes than the controls. The few studies included in the meta-analysis demonstrate that most education methods and forms offer good results and positively affect outcomes. Improved outcomes were found in terms of compliance, self-care, the rehospitalization rate, and knowledge. Masterson Creber et al. reported that motivational interventions are better than traditional education programs that focus on providing didactic information. ${ }^{11}$ The authors emphasized that overall educational approaches for improving self-care in HF had been developed and tested with little impact on HF outcomes. Riegel et al. stated that the motivational approach improves patients' involvement in discussions regarding self-care, while Paradis et al. asserted that it promotes awareness and prepares patients for performing self-care behavior. ${ }^{10,28}$ In the papers that met the inclusion criteria for the present meta-analysis, education was provided in various forms. However, the dominant approaches included face-to-face educational sessions and the use of written materials. Online learning, home visits or phone calls were also used, albeit less commonly. Unfortunately, the variety of educational methods applied precludes the identification of the best approach or the formulation of clear conclusions.

By "motivational interventions", the authors mean counseling focused on cognitive-behavioral therapy and cognitive therapy, whereby the patients' willingness to change their behavior is assessed and strategies are developed to promote efforts to change this behavior in the desired way. Logically, effective self-care may prevent rehospitalization and enhance QoL. In the literature, most studies focus on evaluating the impact of self-care interventions on patientrelated and clinical outcomes, such as self-care behavior, self-efficacy, QoL, exercise, health status, hospitalization, mortality, myocardial stress and systemic inflammation; the impact of education is less commonly investigated. Masterson Creber et al. pointed out that the efficiency of education among HF patients may be limited due to the patients' elderly age, cognitive impairment, drowsiness during the day, poor health awareness, and low motivation. ${ }^{11}$

In the present meta-analysis, self-care improved both in the intervention groups and in the controls, but the improvement was markedly greater in patients targeted by the educational interventions. These findings contribute to the ongoing discussions on the subject. In Masterson Creber et al., the implemented motivational interventions did not improve self-care in relation to routine care, while in Paradis et al., 1 month after motivational interventions including face-to-face meetings and phone calls, there was an improvement in self-efficacy, though not in selfcare maintenance. ${ }^{28,29}$ The differences between the studies may result from differences in intervention duration and follow-up time. Thus, the lack of clear indications from the present meta-analysis may be due to the diverse methodologies in the studies analyzed, the types of education offered and the protocols for evaluating intervention effectiveness. Additionally, the effects of education are influenced by the patients' health literacy and social support. ${ }^{30}$ Researchers indicate that the elderly age of HF patients affects their capacity to obtain, read, understand and process health-related information. ${ }^{31}$

In our analysis, the mean patient age was $66.9 \pm 13.2$ years (range: 45.23-77.6 years). Our research demonstrated that no impact of education on QoL was found in the oldest age 
group, while in younger patients such an impact was indeed found. ${ }^{20,26}$ Many older adults have low levels of health literacy, which results in poor treatment outcome. Additionally, older HF patients are often depressed, which also significantly affects their self-care capabilities. Poor self-care and the presence of depressive symptoms are very common among HF patients. Depression results in low motivation, a lack of enthusiasm and poor adherence to treatment. Insufficient self-care and motivation levels predict lower QoL, frequent rehospitalization and higher mortality. ${ }^{23}$

In our meta-analysis, no significant correlation was found between education and QoL improvement. The studies available consistently show no significant differences in terms of reported QoL between HF patients included in educational interventions and those receiving standard care. In these studies, QoL improves following treatment, regardless of any educational activities. Similar conclusions on the role of education in QoL were reported by Masterson Creber et al., who found that QoL improved both in the intervention group and in the control group, with no statistically significant differences between the 2 groups. ${ }^{11}$ Likewise, in a study by Vaillant-Roussel et al., no differences in terms of QoL improvement were found between patients receiving education and those treated in the standard manner. ${ }^{24}$ Wang et al. and Tung et al. demonstrated positive correlations of QoL with self-care behavior and symptom severity. ${ }^{23,32}$ Greater improvement in self-care behavior was associated with a greater improvement in QoL. In other studies, educational strategies were correlated with QoL benefits, as well as with more satisfaction with treatment and less fatigue. No improvement in terms of exercise was found, which is likely to be due to the old age of the HF patients surveyed. Quality of life in this population can also be expected to change with each patient's clinical condition, symptom severity and ability to cope with the difficulties associated with the illness. Abbasi et al. cite findings from a meta-analysis of 6 papers, demonstrating that a self-management education program resulted in shorter hospital stays, fewer hospitalizations and better adherence to medication in $\mathrm{HF}$ patients. It did not, however, impact mortality, functional abilities, symptoms, or QoL. These differences may result from the variety of interventions, methodologies, educational methods and follow-up durations. ${ }^{26}$

As we have suggested, QoL improvement may depend on a given patient's condition and the type of intervention. We agree that motivational interventions alone are not sufficient to improve the QoL of cardiovascular patients. When studying chronically ill patients, one must consider specific factors affecting their reported QoL, and a possible ceiling effect on how much QoL can improve over time as the disease severity worsens.

In the present meta-analysis, education had the strongest significant impact on adherence to treatment. In this aspect, the published data are consistent, confirming the association between compliance and knowledge. In the meta-analysis by Ruppar et al. on the effectiveness of interventions aimed at improving compliance, an association was indeed found between the effectiveness of supportive activities and adherence levels. ${ }^{33}$ The authors suggested that the observed effects were age-dependent. They also pointed out that motivational interventions were more successful when focusing on just a single effect (in this case compliance) than when addressing additional aspects of health behavior. ${ }^{33}$ Furthermore, the authors emphasize the role of multimorbidity in HF patients as an adverse factor for the effectiveness of any educational intervention. Likewise, they point to potential cultural differences in specific countries and regions, which were not considered in our meta-analysis. ${ }^{33}$

The rehospitalization rate was another outcome investigated here in conjunction with education and intervention effectiveness. However, out of all the studies included in the meta-analysis, only 3 addressed rehospitalization, and therefore further analyses could not be performed. Notably, though, the available data point to a lower number of rehospitalizations as a benefit of the educational interventions undertaken. Clearly, as in the case of the previously discussed aspects, the impact of education on selfcare and symptom monitoring capabilities is a factor.

Promoting knowledge on HF is essential for improving adherence and self-care and for preventing rehospitalization, particularly in the case of HF patients living in rural areas. ${ }^{34,35}$ Some papers have also reported that patient education that increases knowledge about the illness is more effective than other interventions in improving adherence to self-care in HF patients. In this context, Uverzagt et al. and Shah et al. showed that adherence to HF self-care recommendations was affected by the availability of an interdisciplinary team consisting of a nurse, a pharmacist, a dietitian, a social worker, and a physician, as well as by regular follow-up. ${ }^{36,37}$

\section{Conclusions}

Education of patients with HF is a key strategy that has a positive impact on outcomes. The greatest benefit is seen in terms of adherence to pharmaceutical treatment and self-care, while QoL was not associated with education.

There are still too few studies on the impact of education on HF outcomes, hence the need for further studies and for interventions adjusted to patients' specific characteristics.

\section{Practical implications}

Educational interventions for patients with HF should be patient-centered. Motivational interventions significantly improved self-care behavior, adherence, rehospitalization rate, and knowledge levels in HF patients. It is important to asses the level and ability of education. Further studies 
are required to identify the forms of education and the specific behavioral interventions that can successfully improve both clinical and patient-related outcomes in HF.

\section{Study limitations}

One limitation of this meta-analysis was the variety of instruments used to measure patient outcomes in the studies analyzed. Inclusion and exclusion criteria were also varied. Further difficulties were associated with comparing results provided in various units (numerical vs percentage data) or measured at various times after the educational intervention, as well as with the lack of a control group in some of the studies analyzed. Also, the meta-analysis did not include studies measuring adherence to pharmaceutical treatment before and after the educational intervention using a direct method (such as blood sample analysis).

\section{ORCID iDs}

Natalia Alicja Świątoniowska-Lonc

(D) https://orcid.org/0000-0003-4211-9205

Agnieszka Sławuta (D) https://orcid.org/0000-0001-5671-9864

Krzysztof Dudek (D) https://orcid.org/0000-0002-9442-989X

Katarzyna Jankowska (D) https://orcid.org/0000-0002-1437-050X

Beata Katarzyna Jankowska-Polańska

(D) https://orcid.org/0000-0003-1120-3535

\section{References}

1. Ziaeian B, Fonarow GC. Epidemiology and aetiology of heart failure. Nat Rev Cardiol. 2016;13(6):368-378.

2. Cvetinovic N, Loncar G, Farkas J. Heart failure management in the elderly: A public health challenge. Wien Klin Wochenschr. 2016; 128(Suppl 7):466-473.

3. Bui AL, Horwich TB, Fonarow GC. Epidemiology and risk profile of heart failure. Nat Rev Cardiol. 2011;8(1):30-41.

4. Loghmani, L, Monfared MB. The effect of self-care education on knowledge and function of patients with heart failure hospitalized in Kerman city hospitals in 2017. Electron J Gen Med. 2018;15(4):em47.

5. Navidian A, Yaghoubinia F, Ganjali A, Khoshsimaee S. The effect of self-care education on the awareness, attitude, and adherence to self-care behaviors in hospitalized patients due to heart failure with and without depression. PLoS One. 2015;10(6):e0130973. doi:10.1371/ journal.pone.0130973. eCollection 2015.

6. Boyde M, Peters R, New N, Hwang R, Ha T, Korczyk D. Self-care educational intervention to reduce hospitalisations in heart failure: A randomised controlled trial. Eur J Cardiovasc Nurs. 2018;17(2):178-185.

7. Ponikowski P, Voors AA, Anker SD, et al. 2016 ESC guidelines for the diagnosis and treatment of acute and chronic heart failure. Kardiol Pol. 2016;74(10):1037-1147.

8. Jovicic A, Holroyd-Leduc JM, Straus SE. Effects of self-management intervention on health outcomes of patients with heart failure: A systematic review of randomized controlled trials. BMC CardiovasC Disord. 2006;6(1):43.

9. Health education: Theoretical concepts, effective strategies and core competencies. A foundation document to guide capacity development of health educators. http://applications.emro.who.int/dsaf/ EMRPUB_2012_EN_1362.pdf. Accessed June 5, 2019.

10. Riegel B, Dickson VV, Garcia LE, Masterson Creber R, Streur M. Mechanisms of change in self-care in adults with heart failure receiving a tailored, motivational interviewing intervention. Patient Educ Couns. 2017;100(2):283-288.

11. Masterson Creber R, Patey M, Dickson VV, DeCesaris M, Riegel B. Motivational Interviewing Tailored Interventions for Heart Failure (MITI-HF): Study design and methods. Contemp Clin Trials. 2015;41: 62-68.
12. Lutje V. Guide to the search strategy. https://cidg.cochrane.org/sites/ cidg.cochrane.org/files/public/uploads/search-strategy-guide_ modified_8mar2013_1.pdf. Accessed January 2, 2019.

13. Boyne JJ, Vrijhoef HJ, Spreeuwenberg M, De Weerd G, Kragten J, Gorgels AP; TEHAF investigators. Effects of tailored telemonitoring on heart failure patients' knowledge, self-care, self-efficacy and adherence: A randomized controlled trial. Eur J Cardiovasc Nurs. 2014; 13(3):243-252.

14. Deka P, Pozehl B, Williams MA, Norman JF, Khazanchi D, Pathak D. MOVE-HF: An internet-based pilot study to improve adherence to exercise in patients with heart failure. Eur J Cardiovasc Nurs. 2018; 18(2):122-131.

15. Kato NP, Kinugawa K, Sano M, et al. How effective is an in-hospital heart failure self-care program in a Japanese setting? Lessons from a randomized controlled pilot study. Patient Prefer Adherence. 2016; 18(10):171-181.

16. Hägglund E, Lyngå P, Frie F, et al. Patient-centred home-based management of heart failure. Findings from a randomised clinical trial evaluating a tablet computer for self-care, quality of life and effects on knowledge. Scand Cardiovasc J. 2015;49(4):193-199.

17. Kinugasa Y, Kato M, Sugihara S, et al. Multidisciplinary intensive education in the hospital improves outcomes for hospitalized heart failure patients in a Japanese rural setting. BMC Health Serv Res. 2014; 19(14):351.

18. Donner Alves F, Correa Souza G, Brunetto S, Schweigert Perry ID, Biolo A. Nutritional orientation, knowledge and quality of diet in heart failure: Randomized clinical trial. Nutr Hosp. 2012;27(2):441-448.

19. Mussi CM, Ruschel K, de Souza EN, et al. Home visit improves knowledge, self-care and adhesion in heart failure: Randomized clinical trial HELEN-I. Rev Lat Am Enfermagem. 2013;21:20-28.

20. Leventhal ME, Denhaerynck K, Brunner-La Rocca HP, et al. Swiss Interdisciplinary Management Programme for Heart Failure (SWIM-HF): A randomised controlled trial study of an outpatient inter-professional management programme for heart failure patients in Switzerland. Swiss Med Wkly. 2011;8(141):w13171.

21. Ciccone MM, Aquilino A, Cortese F, et al. Feasibility and effectiveness of a disease and care management model in the primary health caresystem for patients with heart failure and diabetes (Project Leonardo). Vasc Health Risk Manag. 2010;6:297-305.

22. Athar, MW, Record JD, Martire C, Hellmann DB, Ziegelstein RC. The effect of a personalized approach to patient education on heart failure self-management. J Pers Med. 2018;8(4). pii: E39. doi:10.3390/ jpm8040039

23. Wang Q, Dong L, Jian Z, Tang X. Effectiveness of a PRECEDE-based education intervention on quality of life in elderly patients with chronic heart failure. BMC Cardiovasc Disord. 2017;17(1):262.

24. Vaillant-Roussel H, Laporte C, Pereira B, et al. Impact of patient education on chronic heart failure in primary care (ETIC): A cluster randomised trial. BMC Fam Pract. 2016;17:80.

25. Korzh O, Krasnokutskiy S. Significance of education and self-management support for patients with chronic heart failure in family physician practice. Family Med Primary Care Rev. 2016;18(4):432-436.

26. Abbasi A, Tahereh NG, Mansoureh AF. Effect of the self-management education program on the quality of life in people with chronic heart failure: A randomized controlled trial. Electron Physician. 2018; 10(7):7028-7037.

27. Higgins JP, Thompson S, Deeks J, Altman DG. Measuring inconsistency in meta-analyses. British Medical Journal. 2003;327:557-560.

28. Paradis V, Cossette S, Frasure-Smith N, Heppell S, Guertin MC. The efficacy of a motivational nursing intervention based on the stages of change on self-care in heart failure patients. J Cardiovasc Nurs. 2010;25(2):130-141.

29. Masterson Creber R, Patey M, Lee CS, Kuan A, Jurgens C, Riegel B. Motivational interviewing to improve self-care for patients with chronic heart failure: MITI-HF randomized controlled trial. Patient Educ Couns. 2016;99(2):256-264.

30. Lee SY, Arozullah AM, Cho YI. Health literacy, social support, and health: A research agenda. Soc Sci Med. 2004;58(7):1309-1321.

31. Wu JR, Moser DK, DeWalt DA, Rayens MK, Dracup K. Health literacy mediates the relationship between age and health outcomes in patients with heart failure. Circ Heart Fail. 2016;9(1):e002250. 
32. Tung HH, Lin CY, Chen KY, Chang CJ, Lin YP, Chou CH. Self-management intervention to improve self-care and quality of life in heart failure patients. Congest Heart Fail. 2013;19(4):E9-E16.

33. Ruppar TM, Delgado JM, Temple J. Medication adherence interventions for heart failure patients: A meta-analysis. Eur J Cardiovasc Nurs. 2015;14(5):395-404.

34. Lee KS, Moser DK, Pelter MM, Nesbitt T, Dracup K. Self-care in rural residents with heart failure: What we are missing. Eur J Cardiovasc Nurs. 2017;16(4):326-333.
35. Dracup K, Moser D, Pelter MM, et al. Rural patients' knowledge about heart failure. J Cardiovasc Nurs. 2014;29(5):423-428.

36. Unverzagt S, Meyer G, Mittmann S, Samos F-A, Unverzagt M, Prondzinsky R. Improving treatment adherence in heart failure: A systematic review and meta-analysis of pharmacological and lifestyle interventions. Dtsch Arztebl Int. 2016;113(25):423-430.

37. Shah D, Simms K, Barksdale D, Wu J-R. Improving medication adherence of patients with chronic heart failure: Challenges and solutions. Res Rep Clin Cardiol. 2015;2015:87-95. 